\title{
YKL-39 (chitinase 3-like protein 2), but not YKL-40 (chitinase 3-like protein 1), is up regulated in osteoarthritic chondrocytes
}

\author{
T Knorr, F Obermayr, E Bartnik, A Zien, T Aigner
}

Objective: To investigate quantitatively the mRNA expression levels of YKL-40, an established marker of rheumatoid and osteoarthritic cartilage degeneration in synovial fluid and serum, and a closely related molecule YKL-39, in articular chondrocytes.

Methods: cDNA array and online quantitative polymerase chain reaction (PCR) were used to measure mRNA expression levels of YKL-39 and YKL-40 in chondrocytes in normal, early degenerative, and late stage osteoarthritic cartilage samples.

Results: Expression analysis showed high levels of both proteins in normal articular chondrocytes, with lower levels of YKL-39 than YKL-40. Whereas YKL-40 was significantly down regulated in late stage osteoarthritic chondrocytes, YKL-39 was significantly up regulated. In vitro both YKLs were down regulated by interleukin $1 \beta$.

Conclusions: The up regulation of YKL-39 in osteoarthritic cartilage suggests that YKL-39 may be a more accurate marker of chondrocyte activation than YKL-40, although it has yet to be established as a suitable marker in synovial fluid and serum. The decreased expression of YKL-40 by osteoarthritic chondrocytes is surprising as increased levels have been reported in rheumatoid and osteoarthritic synovial fluid, where it may derive from activated synovial cells or osteophytic tissue or by increased matrix destruction in the osteoarthritic joint. YKL-39 and YKL-40 are potentially interesting marker molecules for arthritic joint disease because they are abundantly expressed by both normal and osteoarthritic chondrocytes.

B ochemical markers for the osteoarthritic disease process in synovial or other body fluids are greatly needed for patient management. This includes markers for the diagnosis of early osteoarthritis and for monitoring the disease progress, which is central for the development and testing of new therapeutic regimens. This is particularly true as conventional diagnosis by $x$ ray imaging is an insensitive technique and cannot be used to estimate short term developments.

Several authors have reported that chitinase 3-like protein 1 (YKL-40; cartilage glycoprotein 39), originally described as a major gene product of chondrocytes and synovial cells ${ }^{12}$ as well as one component of neutrophil granules, is clinically useful for the diagnosis of osteoarthritic and rheumatoid ${ }^{3-6}$ articular joint disease, being significantly raised in serum and synovial fluid. Contradictory reports exist as to whether normal articular chondrocytes in situ express and secrete YKL-40: thus, by in situ hybridisation no detectable signals were found, ${ }^{7}$ whereas YKL-40 was detectable in normal articular chondrocytes by immunostaining. ${ }^{8}$ Significant increases of mRNA expression levels have been reported for osteoarthritic chondrocytes. ${ }^{179}$
In our recent gene array experiment we suggested that another related molecule, chitinase 3-like protein 2 (YKL-39, chondrocyte protein 39), is up regulated in osteoarthritic chondrocytes. ${ }^{10}$ YKL-39 was originally found to be abundantly secreted by chondrocytes in vitro (about $4 \%$ of all secreted proteins ${ }^{2}$ ). Recently, it has been shown that patients with osteoarthritic or rheumatoid joint disease have autoimmunity against YKL-39. ${ }^{11}$ No studies have yet shown whether this molecule might also be a marker for arthritic joint disease.

In this study, we used cDNA array technology with repetitive probes and online quantitative polymerase chain reaction (PCR) technology to try to answer the question as to whether YKL-40 and YKL-39 are markers of osteoarthritic chondrocytes-that is, whether they show increased expression compared with normal chondrocytes within the (normal and diseased) tissue.

\section{MATERIALS AND METHODS Cartilage samples}

For the study of mRNA expression levels, cartilage from human femoral condyles was processed as described previously. ${ }^{10}$ For the cDNA array (" ${ }_{\text {CDNA }}$ ") and PCR (“" ${ }^{\prime \prime}$ ") experiments normal articular cartilage $\left(\mathrm{n}_{\mathrm{CDNA}}=20 ; 45-88\right.$ years; $\mathrm{n}_{\mathrm{PCR}}=7 ; 32-83$ years) and early degenerated cartilage $\left(\mathrm{n}_{\mathrm{CDNA}}=21 ; 43-91\right.$ years; $\mathrm{n}_{\mathrm{PCR}}=8 ; 43-91$ years $)$ were obtained from necropsies within 48 hours of death. Osteoarthritic cartilage was obtained from total knee replacement $\left(\mathrm{n}_{\mathrm{cDNA}}=20\right.$; 61-84 years; $\mathrm{n}_{\mathrm{PCR}}=8 ; 63-79$ years). Cartilage was considered to be normal if the whole joint showed no significant softening or surface fibrillation of the articular cartilage. Early degenerated cartilage was defined as cartilage derived from a joint which showed overall moderate fibrillation and softening, but no advanced erosion of the articular cartilage. Cases of rheumatoid arthritis were excluded from the study. Only primary degenerated and not regenerative cartilage (osteophytic tissue) was used.

\section{Cell isolation-stimulation with interleukin $1 \beta$ (IL1 $\beta$ )}

For the in vitro culture studies, normal human knee articular cartilage was obtained from normal donors at necropsy within 48 hours of death $(n=3 ; 52-79$ years $)$. Cells were isolated and cultured in short term, high density, monolayer cultures as described previously ${ }^{12}$ and stimulated with 0 (control), l, and $10 \mathrm{ng} / \mathrm{ml}$ recombinant human ILl $\beta$ (Biomol, Germany) with $10 \%$ fetal calf serum (Biochrom, FRG) for three days.

RNA isolation, cDNA synthesis, conventional PCR Total RNA from cartilage tissue and cultured chondrocytes was isolated, and cDNA synthesis and conventional PCR (35

Abbreviations: IL $1 \beta$, interleukin 1 $\beta$; PCR, polymerase chain reaction 
Table 1 Expression differences of YKL-39 and YKL-40 in late stage osteoarthritic compared with normal cartilage according to conventional PCR (cvPCR), quantitative online PCR (qPCR), and cDNA array analysis (array)

\begin{tabular}{llll}
\hline Gene & cvPCR & qPCR & Array \\
\hline YKL-39 & $(\uparrow)$ & $\uparrow(p<0.05)$. & $\uparrow(p<0.02)$ \\
YKL-40 & $(\downarrow)$ & $\downarrow(p<0.01)$ & $\downarrow(p<0.005)$ \\
\hline
\end{tabular}

cycles) performed as described previously. ${ }^{13}$ Three primer pairs were selected using the PRIMER EXPRESS software (Perkin Elmer): YKL40-forward: ATGGGTGTGAAGGCGTCTCAAA CAGG; YKL40-reverse: CTACGTTGCAGCGAGTGCATCCTTG; YKL39-forward GCCGCGACGCGTGACCAGAAGTCTCTCTGGGC; YKL39-reverse: GCCGCGGCGGCCGCCCAAGGAGC CAAGGCTTCTC; actin595.for: GGACCTGGCTGGCCGGGACC; actin1091.rev: AGCCGCCGATCCACACGGAG.

\section{cDNA array analysis}

cDNA arrays were produced with 7808 cDNAs spotted using a custom made needle printer. More than 700 identical arrays have been produced for gene expression studies. Total RNA, I $\mu \mathrm{g}$ from each sample, was labelled with $\left[{ }^{33} \mathrm{P}\right] \mathrm{dCTP}$ and between 0.7 and $1 \times 10^{8}$ counts were hybridised onto four identical arrays using semiautomated hybridisation machines. The reactions were primed using random hexamers. Hybridisations were carried out in plastic boxes in a volume of $60 \mathrm{ml}$ formamide based buffer at $50^{\circ} \mathrm{C}$ overnight.

"Visual Grid" based software was used for image analysis to determine the raw spot intensities.

Local background was determined and the background subtracted from the target spot intensities. Duplicate spots on an array that differed greatly in expression were considered to be outliers and eliminated from further processing. To correct for global differences between arrays, the globalisation method was applied. To consolidate replicate data, the mean and standard error for each target spot were calculated. The mean of the target replicate values was compared with the distribution of the background values, and thus the probability of being significantly expressed was derived for each target.

\section{Quantitative online PCR}

TAQMAN assays were developed and used to detect YKL-39 and YKL-40, as described in detail elsewhere. ${ }^{12}$ The computerselected primers and probes and optimal assay conditions were tested according to the manufacturer's recommendations (Perkin Elmer). The two primer pairs were: YKL39 Taq-Probe ( $100 \mathrm{~nm}$ ) TTGGGAAAAGCCCCTTATCACTG, YKL39Taq forward $(50 \mathrm{~nm})$ CCTCCTGTCCTTTGACTTCCAT, YKL39Taq reverse $(900 \mathrm{~nm})$ CCTTGCTCAGAGGGCTGTTG, YKL40 Taq-Probe $(100 \mathrm{~nm})$ CGGCCAGCAGGTCCCCTATGC, YKL40Tag forward $(50 \mathrm{~nm})$ CGGAGCCACAGTCCATAGAATC, YKL40Tag reverse $(300 \mathrm{~nm}$ ) CGTCGTATCCTACCCACTGGTT.

A separate master mix was made up for each of the primer pairs and contained a final concentration of $200 \mu \mathrm{mol} / \mathrm{l}$ NTPs, $450 \mathrm{nmol} / \mathrm{l}$ Rox buffer, and 100 nmol/l TAQMAN probe. For each gene a standard curve was included using gene-specific standard fragments. ${ }^{12}$ All experiments were performed in triplicate. For standardisation, mRNA ratios relative to glyceraldehyde-3-phosphate dehydrogenase as housekeeping gene were calculated. ${ }^{13}$

\section{Statistical analysis of quantitative PCR}

Statistical evaluation of significant differences in expression levels in vivo was done by the non-parametric WilcoxonMann-Whitney test.
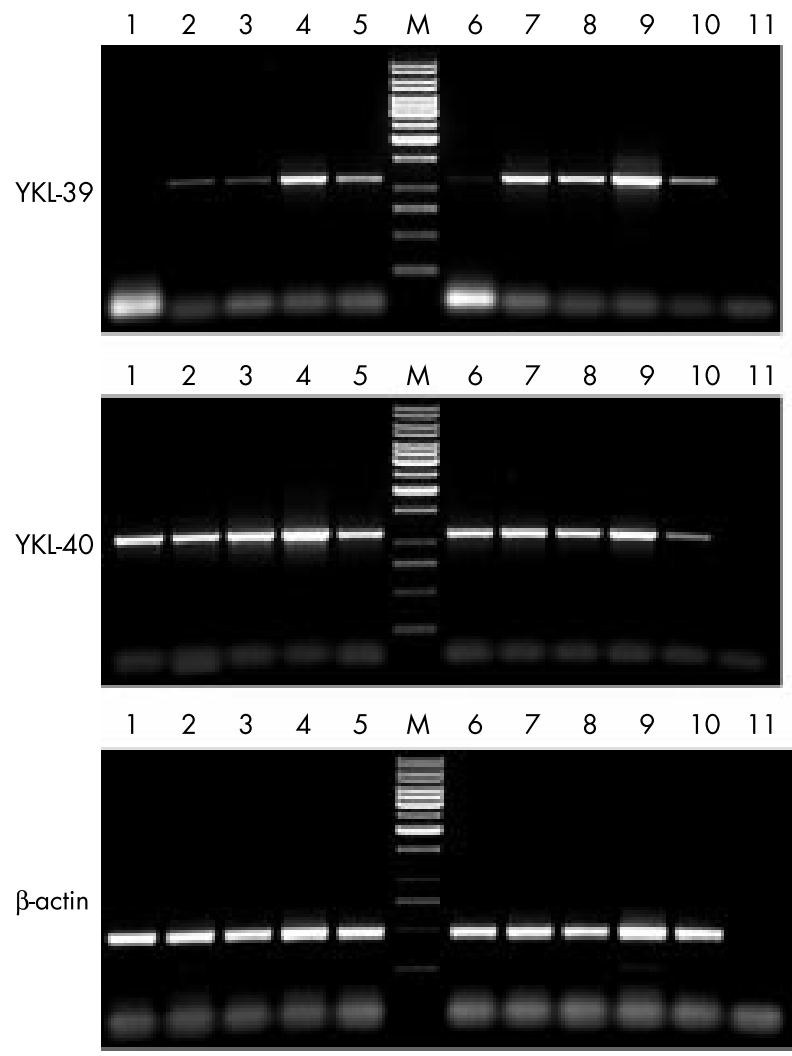

Figure 1 Demonstration of YKL-39, YKL-40, and $\beta$-actin in normal $(\mathrm{n}=5$ : lanes $1-5)$ and osteoarthritic $(\mathrm{n}=5$ : lanes 6-10) articular cartilage by conventional PCR. Lane M, 100 bp DNA ladder (MBI Fermentas, FRG); lane 11, negative (water) control.

\section{Statistical analysis of cDNA array measurements}

Each cDNA array yielded multiple expression values for the investigated genes/proteins, because multiple clones of those genes were spotted onto the arrays. This was used to increase confidence in the analysis results by two strategies: (a) expression change and significance were investigated separately for each clone; $(b)$ a virtual expression profile for the gene was computed by averaging (separately for each array) over the corresponding clones. In both cases, p values were computed with the non-parametric rank sum test (Wilcoxon, Mann-Whitney) and the parametric $t$ test.

\section{RESULTS}

Table l summarises the results obtained.

Conventional reverse transcriptase-PCR analysis of the mRNA expression in normal and osteoarthritic chondrocytes in situ

By conventional PCR mRNA expression of YKL-39 was detectable in most normal $(4 / 5)$ and late stage osteoarthritic (4/5) samples with some indication of up regulation in the latter samples. YKL-40 mRNA was detectable in all samples (five normal and five late stage osteoarthritic), but was somewhat down regulated in late stage osteoarthritic cartilage (fig 1).

\section{Expression analysis by cDNA arrays}

Firstly, we evaluated the expression of both chitinase 3-like molecules using custom made cDNA arrays containing 12 independent spots for YKL-40 and eight independent spots for YKL-39 (four replicates each). This showed high expression levels for YKL-40 in normal articular chondrocytes. Also YKL-39 was highly expressed, though at a significantly lower level than YKL-40 $(\mathrm{p}<0.01)$. A comparison of normal and late 

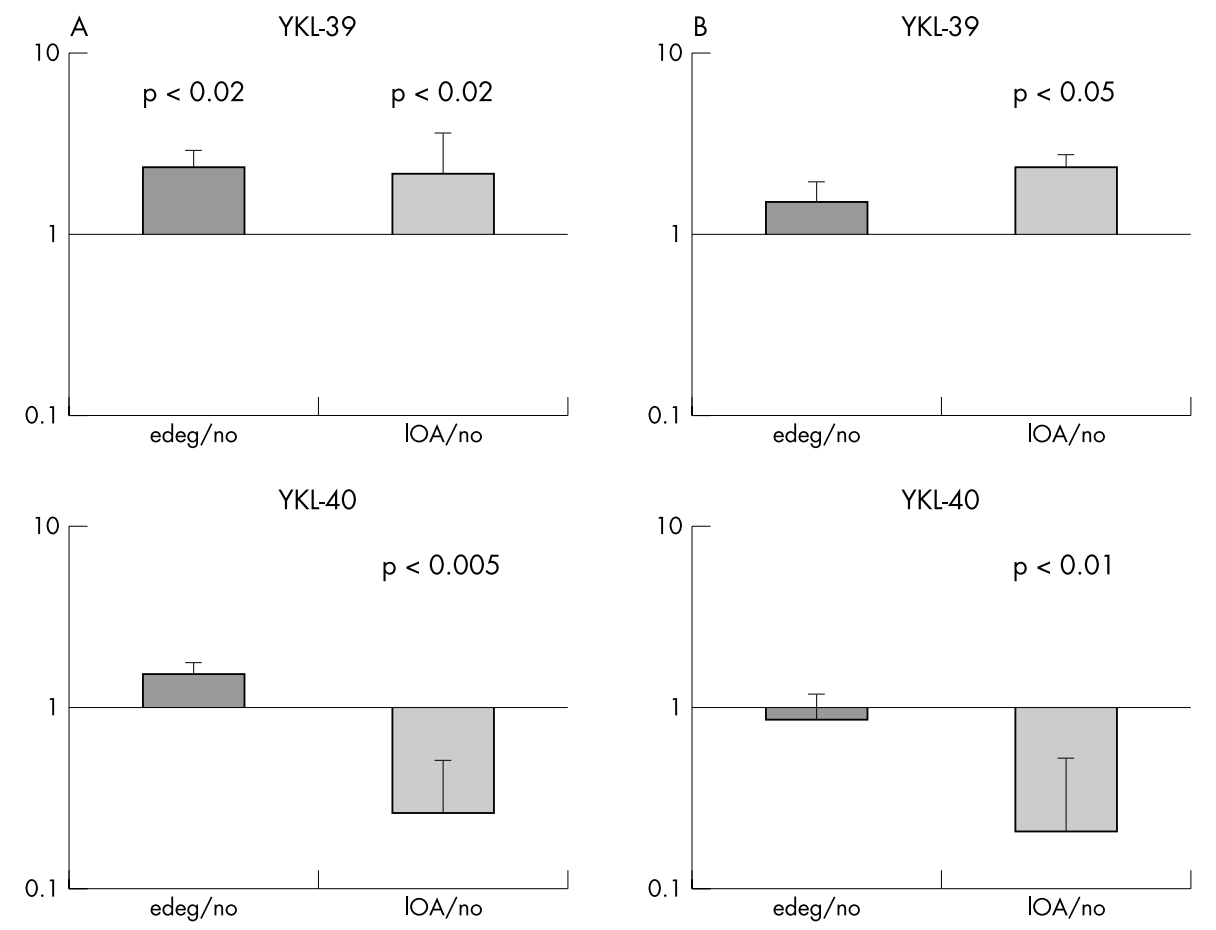

Figure 2 (A) cDNA array analysis of mRNA expression levels in chondrocytes in normal ("no"; $n=20$ ), early degenerative ("edeg"; $n=21$ ), and late stage osteoarthritic ("IOA"; $n=20$ ) articular cartilage for YKL-39 and YKL-40. Results are shown as ratios to normal cartilage. (B) Quantitative TAQMAN analysis for mRNA expression levels of YKL-39 and YKL-40 in normal $(n=7)$, early degenerative $(n=8)$, and late stage osteoarthritic cartilage $(n=8)$. Results are shown as ratios to normal cartilage. Means and standard deviations are shown.

stage osteoarthritic samples showed a significant down regulation of YKL-40 $(\mathrm{p}<0.005)$ and up regulation of YKL-39 $(\mathrm{p}<0.02)$. YKL-39 was also significantly up regulated in early degenerative lesions $(\mathrm{p}<0.02)$ ( fig $2 \mathrm{~A})$.

Quantification of mRNA expression levels in normal and osteoarthritic chondrocytes in vivo and in vitro by TAQMAN analysis

For the quantification of the mRNA levels of YKL-40 and YKL-39 we examined seven normal, eight early degenerative, and eight late stage osteoarthritic cartilage samples. The results confirmed the findings of the cDNA arrays showing that both genes were abundantly expressed in normal and osteoarthritic cartilage with YKL-40 being more abundantly expressed than YKL-39. In late stage osteoarthritic cartilage YKL-40 was down regulated $(\times 4.9 ; \mathrm{p}<0.01)$, whereas YKL-39 was up regulated $(\times 2.4 ; \mathrm{p}<0.05)$ (fig $2 \mathrm{~B})$.

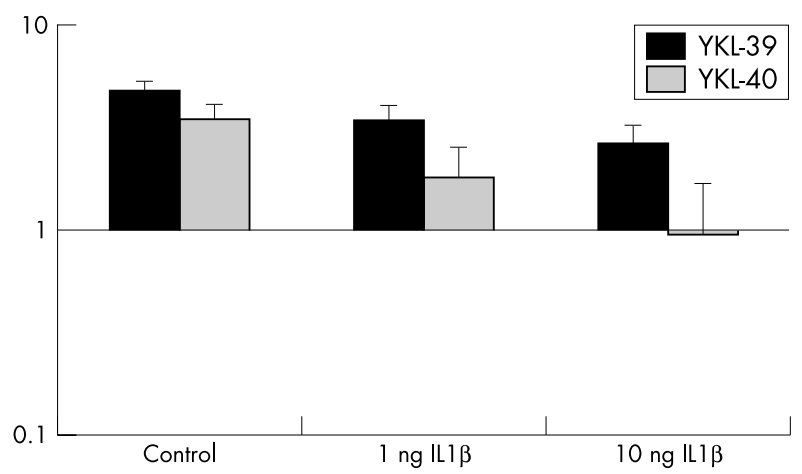

Figure 3 Quantitative TAQMAN analysis for mRNA expression levels of YKL-39 and YKL-40 in isolated normal articular

chondrocytes after stimulation with $1 \mathrm{ng}$ and $10 \mathrm{ng} \mathrm{IL} 1 \beta$. The ratios of IL $1 \beta$ stimulated to non-IL $1 \beta$ stimulated samples in serum-containing media are shown. In all cases three independent experiments were performed.
Quantification of mRNA expression levels in articular chondrocytes in vitro with and without stimulation by IL1 $\beta$

YKL-39 and YKL-40 were slightly more strongly expressed in adult articular chondrocytes in vitro than in vivo. Both YKLS were down regulated by ILl $\beta$ (fig 3).

\section{DISCUSSION}

It has been suggested that YKL-40 may be one of the best candidate biochemical markers for the diagnosis, prognosis, and monitoring of the osteoarthritic and rheumatoid disease process..$^{3-6}$ Our results were unexpected, however, as YKL-40 was expressed at high levels in normal cartilage, but down regulated in osteoarthritic cartilage. This corrects previous reports $^{179}$ in two ways: firstly, normal adult articular chondrocytes do abundantly express YKL-40; secondly, YKL-40 is down regulated and not up regulated in osteoarthritic cartilage. The reason for these discrepancies is somewhat unclear because PCR technology is not needed for abundant gene transcripts such as YKL-40. However, previously used techniques such as in situ hybridisation are always prone to false negative and false positive results.

The increased levels of YKL-40 in osteoarthritic synovial fluid $^{3-5}$ may reflect the increased expression of YKL-40 by other connective tissue cells present within the joints, such as activated synovial fibroblasts or synoviocytes. Indeed, an increased expression of YKL-40 has been shown in rheumatoid synovium. ${ }^{8}$ Also, osteophytic tissue, commonly present in degenerated joints, may significantly contribute to the increased levels of YKL-40 in synovial fluid. ${ }^{7}$ Alternatively, increased levels of YKL-40 in synovial fluid may reflect increased matrix destruction within the osteoarthritic joint, with increased release of YKL-40 from the cartilage matrix into the synovial fluid. This appears to be a realistic suggestion as YKL-40 is presumably abundant in articular cartilage, at least at the molar level, given its high expression in normal articular chondrocytes in situ. 
In contrast with YKL-40, YKL-39 was significantly up regulated in osteoarthritic chondrocytes, in line with our previously reported data. ${ }^{10}$ YKL-39 may, therefore, be a more accurate marker of chondrocyte activation in the disease process, as it was also up regulated in the early degenerative cartilage specimens. However, it has yet to be established as a suitable marker for chondrocyte activation when measured in synovial fluid and serum.

Whereas the function of YKL-39 is not at all clear, YKL-40 appears to have a slight positive effect on proliferation and proteoglycan synthesis of articular chondrocytes and a slight proliferative effect on synoviocytes. ${ }^{14}$ This may suggest that increased synthesis of YKL-40 by the synovial cells leads to further proliferation of this cell population, which is a typical feature of osteoarthritic synoviopathy. ${ }^{15}$ Possibly, YKL-40, diffusing from the synovial fluid into the articular cartilage, contributes to the enhanced proliferative activity of chondrocytes found in osteoarthritic cartilage. ${ }^{16}$ Of note, IL1 $\beta$ down regulated both YKLs, which confirms previous reports on YKL-40. ${ }^{17}$

Overall, YKL-39 and YKL-40 are interesting potential marker molecules for the assessment of arthritic joint disease because they are abundantly expressed by chondrocytes, not only in vitro but also in vivo. However, they are not specific for articular chondrocytes or the osteoarthritic disease process, and YKL-40, in particular, has been shown to be expressed in other organs ${ }^{1}$ and other conditions as well. Interestingly, a prognostic value has been attributed to YKL-40 in many different diseases, including cancer and arteriosclerosis, suggesting it may be a marker of matrix remodelling in a deranged tissue compartment, a situation which occurs in osteoarthritic joints.

\section{ACKNOWLEDGEMENTS}

We are grateful to Freya Boggasch and Anke Nehlen for excellent technical assistance and Brigitte Bau for the help with the TAQMAN experiments.

This work was supported by the German Ministry of Research and Technology (grant 01GG9824).

\section{Authors' affiliations}

T Knorr, T Aigner, Cartilage Research, Department of Pathology, University of Erlangen-Nürnberg, FRG

F Obermayr, GPC Biotech, Munich, FRG

E Bartnik, Aventis Pharma, Frankfurt, FRG

A Zien, Fraunhofer Institut für Algorithmen und Wissenschaftliches

Rechnen, (SCAl), Schloss Birlinghoven, Sankt Augustin, FRG

Correspondence to: Dr T Aigner, Cartilage Research, Department of Pathology, University of Erlangen-Nürnberg, Krankenhausstr 8-10, D-91054 Erlangen, FRG; thomas.aigner@patho.imed.uni-erlangen.de

Accepted 9 February 2003

\section{REFERENCES}

1 Hakala BE, White C, Recklies AD. Human cartilage gp-39, a major secretory product of articular chondrocytes and synovial cells, is a mammalian member of a chitinase protein family. J Biol Chem 1993;268:25803-10

2 Hu B, Trinh K, Figueira F, Price PA. Isolation and sequence of a novel human chondrocyte protein related to mammalian members of the chitinase protein family. J Biol Chem 1993;271:19415-20.

3 Johansen JS, Hvolris J, Hansen M, Backer V, Lorenzen I, Price PA. Serum ykl-40 levels in healthy children and adults. Comparison with serum and synovial fluid levels of $y \mathrm{kl}-40$ in patients with osteoarthritis or trauma of the knee joint. Br J Rheumatol 1996;35:553-9.

4 Conrozier T, Carlier MC, Mathieu P, Colson F, Debard AL, Richard S, et al. Serum levels of YKL-4O and $C$ reactive protein in patients with hip osteoarthritis and healthy subjects: a cross sectional study. Ann Rheum Dis 2000;59:828-31.

5 Kawasaki M, Hasegawa Y, Kondo S, Iwata H. Concentration and localization of YKL-40 in hip joint diseases. J Rheumatol. $2001 ; 28: 341-5$

6 Morgante M, Metelli MR, Morgante D. Observations on the increased serum levels of glycoprotein YKL-40 in patients with rheumatoid arthritis and osteoarthritis. Minerva Med 2001:92:151-3.

7 Connor JR, Dodds RA, Emery JG, Kirkpatrick RB, Rosenberg M, Gowen M. Human articular cartilage glycoprotein 39 (HC gp-39) mRNA expression in adult and fetal chondrocytes, osteoblysts and osteocytes by in situ hybridization. Osteoarthritis Cartilage 2000;8:87-95.

8 Volck B, Johansen JS, Stoltenberg M, Garbarsch C, Price PA, Ostergaard $M$, et al. Studies on YKL-4O in knee joints of patients with rheumatoid arthritis and osteoarthritis. Involvement of YKL-4O in the joint pathology. Osteoarthritis Cartilage 2001:9:203-14.

9 Volck B, Ostergaard K, Johansen JS, Garbarch C, Price PA. The distribution of YKL-40 in osteoarthritic and normal human articular cartilage. Scand J Rheumatol 1999;28:171-9.

10 Aigner T, Zien A, Gehrsitz A, Gebhard PM, McKenna LA. Anabolic and catabolic gene expression pattern analysis in normal versus osteoarthritic cartilage using complementary DNA-array technology. Arthritis Rheum 2001;44:2777-89.

11 Tsuruha J, Masuko-Hongo K, Kato T, Sakata M, Nakamura H, Sekine T, et al. Autoimmunity against YKL-39, a human cartilage derived protein, in patients with osteoarthritis. J Rheumatol 2002;29:1459-66.

12 Bau B, Gebhard PM, Haag J, Knorr T, Bartnik E, Aigner T. Relative messenger RNA expression profiling of collagenases and aggrecanases in human articular chondrocytes in vivo and in vitro. Arthritis Rheum 2002;46:2648-57.

13 McKenna LA, Gehrsitz A, Soeder S, Eger W, Kirchner T, Aigner T. Effective isolation of high quality total RNA from human adult articular cartilage. Anal Biochem 2000;286:80-5.

14 De Ceuninck F, Gaufillier S, Bonnaud A, Sabatini M, Lesur C, Pastoureau P. YKL-40 (cartilage gp-39) induces proliferative events in cultured chondrocytes and synoviocytes and increases glycosaminoglycan synthesis in chondrocytes. Biochem Biophys Res Commun 2001;285:926-31.

15 Oehler S, Neureiter D, Meyer-Scholten C, Aigner T. Subtyping of osteoarthritic synoviopathy. Clin Exp Rheumatol 2002;20:633-640.

16 Aigner T, Hemmel M, Neureiter D, Gebhard PM, Zeiler G, Kirchner T, et al. Apoptotic cell death is not a widespread phenomenon in normal aging and osteoarthritic human articular knee cartilage: a study of proliferation, programmed cell death (apoptosis), and viability of chondrocytes in normal and osteoarthritic human knee cartilage. Arthritis Rheum 2001;44:1304-12.

17 Johansen JS, Olee T, Price PA, Hashimoto S, Ochs RL, Lotz M. Regulation of YKL-40 production by human articular chondrocytes. Arthritis Rheum 2001;44:826-37. 